\title{
A two-stage stochastic transportation problem with fixed handling costs and a priori selection of the distribution channels
}

\author{
Yolanda Hinojosa • Justo Puerto • \\ Francisco Saldanha-da-Gama
}

Received: 11 January 2013 / Accepted: 28 January 2014 / Published online: 6 March 2014

(C) Sociedad de Estadística e Investigación Operativa 2014

\begin{abstract}
In this paper, a transportation problem comprising stochastic demands, fixed handling costs at the origins, and fixed costs associated with the links is addressed. It is assumed that uncertainty is adequately captured via a finite set of scenarios. The problem is formulated as a two-stage stochastic program. The goal is to minimize the total cost associated with the selected links plus the expected transportation and fixed handling costs. A prototype problem is initially presented which is then progressively extended to accommodate capacities at the origins and multiple commodities. The results of an extensive set of computational tests are reported and discussed.
\end{abstract}

Keywords Transportation - Stochastic demands · Fixed handling costs · Two-stage stochastic programming

Mathematics Subject Classification (2010) 90B06 · 90B15 · 90C15

\section{Introduction}

In a transportation problem, some commodity available at a set of origins must be shipped to a set of destinations. For each origin-destination pair, there is a unitary cost

\footnotetext{
Y. Hinojosa

Departamento de Economía Aplicada I, Universidad de Sevilla, Sevilla, Spain

J. Puerto

Departamento Estadística e Investigación Operativa, Facultad de Matemáticas, Universidad de Sevilla, Sevilla, Spain

F. Saldanha-da-Gama ( $\varangle)$

DEIO-CIO, Faculdade de Ciências, Universidade de Lisboa, Lisboa, Portugal e-mail: fsgama@fc.ul.pt
} 
for the flow shipped through the corresponding link. The goal is to find the distribution pattern that minimizes the overall transportation cost.

One common assumption in the literature is that every origin-destination pair represents a possible channel for shipping the commodity. In addition, it is often considered that such channels are available when necessary. However, in many situations, none of the previous conditions hold. One can find examples in logistics systems in which not only are some distribution channels infeasible but also some contract (e.g., an option) must be celebrated before setting a (feasible) channel ready for being used (see, for instance, Xu and Nozick 2009). Other examples can be found in telecommunications where traffic can only be sent through some channel if such channel has been previously made physically available (see Ahuja et al. 1993). Finally, a classical example arises in production planning when a set of machines is available during a certain period of time for producing a set of products (not all machines necessarily being able to produce all products) and one has to decide which machines will produce which products and in what quantities (see, for instance, Grieco et al. 2001 and the references therein).

Another frequent assumption in a transportation problem is that the commodity to be shipped is readily available at the origins. Again, this is not always the case. Often the commodity has to be packed or handled somehow before being shipped. This may imply fixed and/or variable costs (e.g., the preparation of some specific equipment). Variable handling costs at the origins can be easily embedded in the variable transportation costs. However, fixed handling costs have to be explicitly considered in a model.

Finally, in many transportation problems, it is assumed that demand is deterministic and known in advance. However, nowadays, due to many factors (e.g., the effect of marketing campaigns, the evolution of the global economy, the appearance of new competitors, etc.) markets tend to become extremely volatile leading to continuously changing demands, which often occurs in an unpredictable manner.

In this paper, a transportation problem is proposed which gathers in the same modeling framework the three features mentioned above. In particular, it is assumed that a strategic (tactical) decision has to be made here-and-now concerning the distribution channels that should be selected/contracted/activated for shipping the commodity. Such decision is made before knowing the exact demand that will occur. After demand is disclosed, the tactical (operational) decision corresponding to the transportation pattern is determined and the final costs can be evaluated. The second-stage decision is constrained by the distribution channels selected/contracted/activated a priori. Fixed costs are considered for the distribution channels (for instance, due to an option contract). Furthermore, variable transportation costs (possibly including variable handling costs at the origins) are considered as well as fixed handling costs at the origins. As mentioned above, the latter may correspond, for example, to the set-up or preparation of some equipment for processing or handling the commodity. The problem is formulated as a two-stage stochastic mixed $0-1$ problem. In the first stage, the decision to be made regards the here-and-now decision about the distribution channels to select; in the second stage - once the demand is known - the transportation decisions are made and the transportation and handling costs are accounted for. 
Demand is assumed to be described by a discrete random vector, with a finite domain, following a probability distribution that is known in advance. In other words, uncertainty is assumed to be well captured by a finite set of scenarios, each of which with some known probability of occurrence.

Despite being of practical relevance, not much literature can be found on stochastic transportation problems. One of the earliest works is due to Williams (1963). A twostage stochastic programming problem is considered. In the first stage (before demand is known) a transportation decision is made. This decision may result in under or oversupply, which is accounted for in the second stage. In particular, penalty costs (for unsupplied demand) and salvage costs (for oversupply) are considered. The problem consists of finding the transportation plan that minimizes the total transportation costs plus the expected costs for under and oversupply. The same problem has been addressed by other authors (see Holmberg 1995; Holmberg and Jörnsten 1984; Qi 1985, and the references therein).

The extension of the problem in which a fixed cost is associated with the operating origins is the so-called stochastic transportation-location problem and has been addressed by França and Luna (1982), Holmberg and Tuy (1999), and LeBlanc (1977). The problem consists of deciding the facilities to locate and the amounts to ship from each operating facility to the customers before knowing the demand. The goal is to minimize the total location and transportation costs plus the expected cost for shortages and surplus.

Max Shen et al. (2003) extend the stochastic transportation-location problem by considering inventory decisions at the destinations. Risk pooling benefits are obtained by considering some destinations as redistribution points which allows safety stocks to be considered and consequently, a pre-specified service level to be achieved.

Another extension of the 'classical' stochastic transportation problem was addressed by Barbarosoğlu and Arda (2004) in the context of disaster response. A two-stage stochastic network flow problem is considered with several transportation modes. Uncertainty is considered not only for the demand but also for the supply capacity and route capacities. The goal is to minimize the expected inventory, transportation, surplus and shortage costs.

A different perspective on transportation problems under uncertainty is considered by Tsai et al. (2011), who consider a transportation problem with uncertainty in the transportation capacities and costs.

Lium et al. (2009) find consolidation as a way to hedge against uncertain demand in a multi-commodity stochastic service network design model. The two-stage stochastic problem considered involves the choice of a service frequency (in the first stage) and the decision about the transportation quantities (in the second stage). The goal is to minimize the total cost associated with the vehicles plus the expected cost for distributing the (multiple) commodities.

More recently, Thapalia et al. (2012) address a stochastic network flow problem in which, a decision has to be made about the edges that should operate as well as their capacity level to cope with stochastic demand. The goal is to minimize the total cost associated with the edges plus the total expected transportation, shortage and salvage costs. The same authors (Thapalia et al. 2012) consider random capacities in a single-commodity network design problem. 
Finally, we can point out the recent work by Li et al. (2012) who consider a location distribution problem with uncertain demand. Uncertainty, driven by a catastrophe such as a hurricane, is captured via a finite set of scenarios.

At a first glance one could think that the problem we address in this paper is simply a location-transportation problem with stochastic demands. However, this is not the case. First, the decision about which origins to "activate" is now a recourse decision. Moreover, as it will be shown later, the setting considered in this paper changes the structure of the solutions that is typically associated with the stochastic locationtransportation problem.

The problem at hand can be put in the context of stochastic network design problems. Nevertheless, it corresponds to a new paradigm which, to the best knowledge of the authors, has not been considered so far.

Our contribution with this paper is twofold: first, to propose a modeling framework for integrating all the aspects discussed above; second, to assert the extent to which an off-the-shelf commercial solver can be used to solve the problem to optimality using the models proposed.

The remainder of the paper is organized as follows. In Sect. 2, the base setting of the problem is introduced and some modeling issues are discussed. In the following section, this setting is extended with some features that are relevant from a practical point of view. In Sect. 4, we report the computational experiments performed with the models discussed. The paper ends with some conclusions drawn from the work present and some ideas for further research.

\section{Problem formulation}

As mentioned above, we address a two-stage transportation problem with stochastic demands. Before demand is disclosed, a decision has to be made regarding the distribution channels/links that should be selected. For each selected link, a fixed set-up cost is incurred. After demand is disclosed, a recourse decision is considered which consists in defining the quantities to ship from the origins to the destinations using the channels previously selected. A cost is considered for each unit of flow traversing a link. A fixed handling cost is considered at each operating origin.

The fact that the decision about the distribution channels to set-up is made hereand-now (i.e., before knowing the demand) may lead to setting up some links which in the end are not used due to short demand. Nevertheless, like in many investment problems, some risk has to be incurred. Such risk is somehow accounted for by the trade-off imposed in the model we propose below for all costs involved in the problem.

To formulate the problem, the notation below is considered. Sets:

I Set of origins.

$J$ Set of destinations.

Deterministic parameters:

$c_{i j}$ Set-up/activation cost for link $(i, j), i \in I, j \in J$.

$d_{i j}$ Unitary cost for the flow in arc $(i, j), i \in I, j \in J$.

$h_{i}$ Fixed handling cost at origin $i \in I$. We assume that this value is strictly positive. 
Stochastic parameters:

$D_{j}$ Demand of customer $j \in J$.

The decisions to be made can be represented by the following decision variables:

$$
y_{i j}=\left\{\begin{array}{ll}
1 & \text { if link }(\mathrm{i}, \mathrm{j}) \text { is selected } \\
0 & \text { otherwise }
\end{array} \quad i \in I, j \in J\right.
$$

$x_{i j}=$ Flow to be shipped from $i \in I$ to $j \in J$.

$$
z_{i}=\left\{\begin{array}{ll}
1 & \text { if origin i is used } \\
0 & \text { otherwise }
\end{array} \quad i \in I .\right.
$$

The problem can be formulated as follows:

$$
\begin{array}{ll}
\min & \sum_{i \in I} \sum_{j \in J} c_{i j} y_{i j}+\mathcal{Q}(\mathbf{y}) \\
\text { s.t. } & \sum_{i \in I} y_{i j} \geq 1 \quad j \in J \\
y_{i j} \in\{0,1\} \quad i \in I, j \in J
\end{array}
$$

with $\mathcal{Q}(\mathbf{y})=E[Q(\mathbf{y})]$ and

$$
\begin{gathered}
Q(\mathbf{y})=\min \quad \sum_{i \in I} \sum_{j \in J} d_{i j} x_{i j}+\sum_{i \in I} h_{i} z_{i} \\
\text { s.t. } x_{i j} \leq M y_{i j} \quad i \in I, j \in J \\
\sum_{i \in I} x_{i j} \geq D_{j} \quad j \in J \\
\sum_{j \in J} x_{i j} \leq M z_{i} \quad i \in I \\
\sum_{j \in J} y_{i j} \geq z_{i} \quad i \in I \\
x_{i j} \geq 0 \quad i \in I, j \in J \\
z_{i} \in\{0,1\} \quad i \in I
\end{gathered}
$$

The first-stage problem determines the distribution channels to be selected. The objective function (1) evaluates the total fixed cost for such links. Constraints (2) assure that each customer has at least one distribution channel available for receiving the commodity. Constraints (3) define the domain of the network design variables.

In the second stage, the flows are determined. The objective function (4) quantifies the total distribution cost (possibly including variable handling costs at the origins) and the total fixed handling costs at the origins. Constraints (5) assure that flow can only be shipped through the channels previously selected. $M$ denotes a large value. 
Constraints (6) assure the satisfaction of the demand. Constraints (7) assure that if flow is shipped from some origin then the corresponding fixed handling cost must be accounted for. As above, $M$ denotes a large value. Constraints (8) ensure that origin $i$ can be activated only if some link $(i, j)$ is set-up. Finally, constraints (9) and (10) are domain constraints.

Note that in the second-stage problem, variables $y_{i j}$ are fixed and $D_{j}$ are random variables. Furthermore, the coefficients of the $y$-variables in the second-stage problem are deterministic. Therefore, the problem has fixed recourse.

Remark 1 Due to the fact that no capacity constraint is considered at the origins, once at least one distribution channel exists for each customer (which is assured by the first-stage problem), there is always a feasible solution to the second-stage problem. Accordingly, this is a stochastic problem with (relatively) complete recourse. Note that the problem would be of no interest if constraints (2) were not considered. By these constraints, the decision maker is explicitly assuring that the problem is of relevance to $\mathrm{him} / \mathrm{her}$.

\subsection{Extensive form of the deterministic equivalent}

If demand levels are fully determined by a finite range of levels, say $S$, it is possible to go further in terms of the problem formulation. With this purpose, define

$D_{j s} \quad$ Demand level of customer $j \in J$ under demand level $s$.

$p_{s} \quad$ Probability that demand level $s$ occurs.

Consider also the following redefinition of the recourse decision variables:

$x_{i j s}=$ Flow to be shipped from $i \in I$ to $j \in J$ under demand level $s \in S$.

$$
z_{i s}=\left\{\begin{array}{ll}
1 & \text { if origin i handles flow in demand level s } \\
0 & \text { otherwise }
\end{array} \quad i \in I, s \in S .\right.
$$

The extensive form of the deterministic equivalent is as follows:

$$
\begin{array}{ll}
\min & \sum_{i \in I} \sum_{j \in J} c_{i j} y_{i j}+\sum_{s \in S} p_{s}\left[\sum_{i \in I} \sum_{j \in J} d_{i j} x_{i j s}+\sum_{i \in I} h_{i} z_{i s}\right] \\
\text { s.t. } & \sum_{i \in I} y_{i j} \geq 1 \quad j \in J \\
& x_{i j s} \leq M y_{i j} \quad i \in I, j \in J, s \in S \\
& \sum_{i \in I} x_{i j s} \geq D_{j s} \quad j \in J, s \in S \\
& \sum_{j \in J} x_{i j s} \leq M z_{i s} \quad i \in I, s \in S \\
& \sum_{j \in J} y_{i j} \geq z_{i s} \quad i \in I, s \in S
\end{array}
$$




$$
\begin{aligned}
& y_{i j} \in\{0,1\} \quad i \in I, j \in J \\
& x_{i j s} \geq 0 \quad i \in I, j \in J, s \in S \\
& z_{i s} \in\{0,1\} \quad i \in I, s \in S
\end{aligned}
$$

It should be pointed out that first-stage variables $y$ are the same for all demand levels, which means that the non-anticipativity principle is being implicitly considered in the above formulation. Variables $x$ and $z$ are the second-stage decision variables. In this model, the first stage contains only binary variables whereas the second stage contains binary and continuous variables.

Note that constraints (13) and (15) can be "tuned" by considering $M=D_{j s}$ in (13) and $M=\sum_{j \in J} D_{j s}$ in (15).

In addition, the reader should also observe that constraints (12) are redundant in this model as they appear together with constraints (13) and (14).

Finally, the objective function together with constraints (13) and (15) assure that constraints (16) hold: by constraints (15), $z_{i s}=1$ if and only if $x_{i j s}>0$ for some $j$ (note that, since the term $h_{i} z_{i s}>0$ appears in the objective function, if $x_{i j s}=0$ for all $j$, then $z_{i s}=0$ ). On the other hand, if $x_{i j s}>0$, then $y_{i j}=1$ by constraints (13). Thus, constraints (16) hold and they can be removed from the model.

Remark 2 No capacity constraints are considered in the problem above. Accordingly, one might think that the commodity is always shipped via the cheapest activated links regardless of the demand level defined by the different scenarios. If this was the case, the problem could be reduced to the standard simple plant location problem. However, this is not the case. In fact, to hedge against uncertainty, it may be convenient to select more links, even though some of them will not be used in all scenarios, if the variation in the demand levels is large enough to compensate the activation costs. The following example highlights this aspect.

Consider an instance of the problem with $|I|=2$ origins, $|J|=3$ destinations, and $|S|=2$ demand levels (scenarios). Furthermore, consider the parameters given as follows:

- $c_{i j}=1$ for all $(i, j), i \in I, j \in J$.

- $h_{i}=4$ for all $i \in I$.

- $d_{i j}$ for all $(i, j), i \in I, j \in J$, are given according to the following table:

\begin{tabular}{llll}
\hline & $j_{1}$ & $j_{2}$ & $j_{3}$ \\
\hline$i_{1}$ & 3 & 3 & 1 \\
$i_{2}$ & 1 & 1 & 2 \\
\hline
\end{tabular}

- $D_{j s}$ for all $j \in J, s \in S$, are given by:

\begin{tabular}{llll}
\hline & $j_{1}$ & $j_{2}$ & $j_{3}$ \\
\hline$s_{1}$ & 2 & 2 & 1 \\
$s_{2}$ & 1 & 1 & 10 \\
\hline
\end{tabular}

- $p_{s}=0.5$ for all $s \in S$. 
(a)

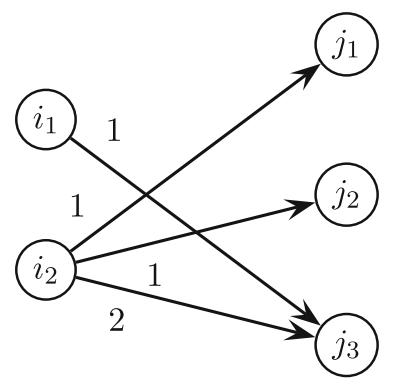

(b)

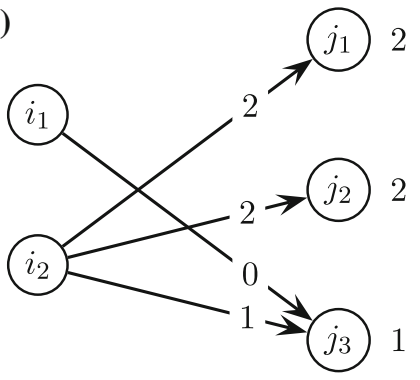

(c)

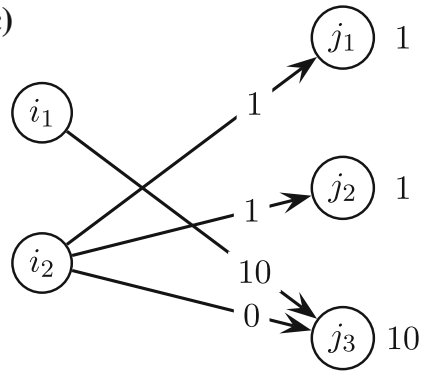

Fig. 1 Example. a Distribution channels selected with the unitary transportation costs. b Scenario 1: transportation decisions. c Scenario 2: transportation decisions

Figure 1 illustrates the optimal solution (which is straightforward to obtain). In particular, Fig. 1a depicts the distribution channels selected and the corresponding unitary transportation cost. In scenario 1 (Fig. 1b) only origin $i_{2}$ is used to serve all destinations, whereas in scenario 2 (Fig. 1c), $j_{1}$ and $j_{2}$ are served by origin $i_{2}$ and $j_{3}$ is served by origin $i_{1}$. It is easy to conclude that the optimal value for the objective function is 19 .

As it can be observed, in scenario $1, \operatorname{link}\left(i_{2}, j_{3}\right)$ is used although it is not the cheapest available in terms of the unitary transportation costs for shipping to destination $j_{3}$. This is explained by the existence of fixed handling costs at the origins, which makes it less attractive to use origin $i_{1}$. Furthermore, in this example, we can see that it may be optimal to set up some arc that is not used in all scenarios.

\section{Extensions of the base setting}

In this section, we progressively extend the basic setting introduced in Sect. 2 to incorporate two features of practical relevance: the existence of multiple commodities and capacities at the origins. These extensions will be defined, giving rise to the corresponding stochastic problems.

\subsection{Multiple commodities}

One immediate extension of the problem regards the case in which there are multiple commodities. Consider the following notation: 
$L \quad$ Set of commodities

$d_{i j}^{\ell} \quad$ Unitary cost for the flow of commodity $\ell \in L$ in $\operatorname{arc}(i, j), i \in I, j \in J$.

$h_{i}^{\ell} \quad$ Fixed handling cost for commodity $\ell \in L$ at origin $i \in I$.

$D_{j}^{\ell} \quad$ (Stochastic) Demand of customer $j \in J$ for commodity $\ell \in L$.

To formulate the problem we consider the first-stage $y$-variables defined as before.

Regarding the recourse decisions, we now consider:

$x_{i j}^{\ell}=$ Flow of commodity $\ell \in L$ to be shipped from $i \in I$ to $j \in J$.

$$
z_{i}^{\ell}=\left\{\begin{array}{ll}
1 & \text { if origin } \mathrm{i} \text { is used for shipping commodity } \ell \\
0 & \text { otherwise }
\end{array} \quad i \in I, \ell \in L .\right.
$$

The first-stage problem is (1)-(3) as before. Regarding the second-stage problem, we now have:

$$
\begin{aligned}
Q(\mathbf{y})=\min & \sum_{i \in I} \sum_{j \in J} \sum_{\ell \in L} d_{i j}^{\ell} x_{i j}^{\ell}+\sum_{i \in I} \sum_{\ell \in L} h_{i}^{\ell} z_{i}^{\ell} \\
\text { s.t. } & \sum_{\ell \in L} x_{i j}^{\ell} \leq M y_{i j} \quad i \in I, j \in J \\
& \sum_{i \in I} x_{i j}^{\ell} \geq D_{j}^{\ell} \quad j \in J, \ell \in L \\
& \sum_{j \in J} x_{i j}^{\ell} \leq M z_{i}^{\ell} \quad i \in I, \ell \in L \\
& x_{i j}^{\ell} \geq 0 \quad i \in I, j \in J, \ell \in L \\
& z_{i}^{\ell} \in\{0,1\} \quad i \in I, \quad \ell \in L
\end{aligned}
$$

The objective function (20) and constraints (21)-(25) have the same meaning as (4) and (5)-(10), respectively, but now adapted to the multi-commodity setting.

As before, variables $y_{i j}$ are fixed, $D_{j}^{\ell}$ are random variables, and the problem has (relatively) complete recourse.

If demand levels are fully determined by the finite set of demand levels $S$, we consider $D_{j s}^{\ell}$ denoting the demand level of customer $j \in J$ for commodity $\ell$ under scenario $s \in S$. In addition, we define:

$x_{i j s}^{\ell}=$ Flow of commodity $\ell$ to be shipped from $i \in I$ to $j \in J$ under a demand level $s \in S$.

$z_{i s}^{\ell}=\left\{\begin{array}{ll}1 & \text { if origin i handles the commodity } \ell \text { in scenarios } \\ 0 & \text { otherwise }\end{array} \quad i \in I, \ell \in L, s \in S\right.$.

The extensive form of the deterministic equivalent can be written as follows:

$$
\min \sum_{i \in I} \sum_{j \in J} c_{i j} y_{i j}+\sum_{s \in S} p_{s}\left[\sum_{i \in I} \sum_{j \in J} \sum_{\ell \in L} d_{i j}^{\ell} x_{i j s}^{\ell}+\sum_{i \in I} \sum_{\ell \in L} h_{i}^{\ell} z_{i s}^{\ell}\right]
$$




$$
\begin{array}{ll}
\text { s.t. } & \sum_{i \in I} y_{i j} \geq 1 \quad j \in J \\
& \sum_{\ell \in L} x_{i j s}^{\ell} \leq \sum_{\ell \in L} D_{j s}^{\ell} y_{i j} \quad i \in I, j \in J, s \in S, \\
& \sum_{i \in I} x_{i j s}^{\ell} \geq D_{j s}^{\ell} \quad j \in J, \ell \in L, s \in S \\
& \sum_{j \in J} x_{i j s}^{\ell} \leq z_{i s}^{\ell} \sum_{j \in J} D_{j s}^{\ell} \quad i \in I, \ell \in L, s \in S . \\
& y_{i j} \in\{0,1\} \quad i \in I, j \in J \\
& x_{i j s}^{\ell} \geq 0 \quad i \in I, j \in J, \ell \in L, s \in S \\
& z_{i s}^{\ell} \in\{0,1\} \quad i \in I, \ell \in L, s \in S
\end{array}
$$

Note that in the above formulation, constraints (28) and (30) represent already a refinement in which " $M$ " was replaced by more appropriate constants.

\subsection{Introduction of capacity constraints}

So far we have assumed that no limit exists on the amounts to be shipped through the network. However, in practice, this is often not the case. In fact, even when no capacities exist on the links, one may find limited capacity at the origins (e.g., handling capacity). Taking this aspect into account, we assume now that in addition to multiple commodities, there are supplying capacities at the origins. In particular, for each $i \in I$ and $\ell \in L$ denote by $K_{i}^{\ell}$ the maximum amount of commodity $\ell$ available at origin $i$. The capacitated problem can be easily formulated by replacing (23) with

$$
\sum_{j \in J} x_{i j}^{\ell} \leq K_{i}^{\ell} z_{i}^{\ell} \quad i \in I,
$$

or by replacing (30) with

$$
\sum_{j \in J} x_{i j s}^{\ell} \leq K_{i}^{\ell} z_{i s}^{\ell} \quad i \in I, s \in S
$$

The introduction of the previous capacity constraints has an impact on the feasibility structure of the problem: it may happen that a first-stage solution (the selected distribution channels) is such that for some scenarios no feasible solution exists because the total available capacity at the origins to which the customers are connected is not enough to satisfy the demand. This means that the (relatively) complete recourse property is lost. Such type of first-stage solution is undesirable. This can be explicitly stated in the model by considering a penalty cost for non-supplied demand, which makes sense from a practical point of view (e.g., non-supplied demands means an opportunity loss that often can be accounted for monetarily). This penalty can only be evaluated after demand is known which means that it should force the second-stage 
problem to comprise the new cost component. In particular, the second-stage function $Q(\mathbf{y})$ can be extended by considering one additional term representing this penalty.

Denote by $\psi_{j}^{\ell}$ the cost per unit of demand of customer $j$ in terms of commodity $\ell$ that is not supplied. In addition, let $\Lambda_{j}^{\ell}$ be the amount of demand which is not supplied. The second-stage problem becomes

$$
\begin{array}{ll}
Q(\mathbf{y})=\min & \sum_{i \in I} \sum_{j \in J} \sum_{l \in L} d_{i j}^{\ell} x_{i j}^{\ell}+\sum_{i \in I} \sum_{l \in L} h_{i}^{\ell} z_{i}^{\ell}+\sum_{j \in J} \sum_{l \in L} \psi_{j}^{\ell} \Lambda_{j}^{\ell} \\
\text { s.t. } & \sum_{\ell \in L} x_{i j}^{\ell} \leq M y_{i j} \quad i \in I, j \in J \\
& \Lambda_{j}^{\ell} \geq D_{j}^{\ell}-\sum_{i \in I} x_{i j}^{\ell} \quad j \in J, \ell \in L \\
& \sum_{j \in J} x_{i j}^{\ell} \leq K_{i}^{\ell} z_{i}^{\ell} \quad i \in I, \ell \in L \\
& \Lambda_{j}^{\ell} \geq 0 \quad j \in J, \ell \in L \\
& x_{i j}^{\ell} \geq 0 \quad i \in I, j \in J, \ell \in L \\
& z_{i}^{\ell} \in\{0,1\} \quad i \in I, \ell \in L
\end{array}
$$

The magnitude of $\psi_{j}^{\ell}$ compared with the costs $d_{i j}^{\ell}$ and $h_{i}^{\ell}$ will be crucial to define the extent to which the decision maker accepts not to supply demand. For $\psi_{j}^{\ell}>\max _{i \in I} d_{i j}^{\ell}+\frac{\max _{i \in I}\left\{c_{i j}+h_{i}^{\ell}\right\}}{D_{j}^{\ell}}$ the demand will be supplied until the limit of the operating distribution capacity. In any case, the problem has now (relatively) complete recourse as there is no obligation to supply all the demand. Therefore, the second-stage feasibility set is always non-empty.

Finally, the extensive form of the deterministic equivalent becomes:

$$
\begin{aligned}
& \min \sum_{i \in I} \sum_{j \in J} c_{i j} y_{i j}+\sum_{s \in S} p_{s}\left[\sum_{i \in I} \sum_{j \in J} \sum_{\ell \in L} d_{i j}^{\ell} x_{i j s}^{\ell}+\sum_{i \in I} \sum_{\ell \in L} h_{i}^{\ell} z_{i s}^{\ell}+\sum_{j \in J} \sum_{\ell \in L} \psi_{j}^{\ell} \Lambda_{j s}^{\ell}\right] \\
& \text { s.t. } \quad \sum_{i \in I} y_{i j} \geq 1 \quad j \in J \\
& \sum_{\ell \in L} x_{i j s}^{\ell} \leq \sum_{\ell \in L} D_{j s}^{\ell} y_{i j} \quad i \in I, j \in J, s \in S \\
& \Lambda_{j s}^{\ell} \geq D_{j s}^{\ell}-\sum_{i \in I} x_{i j s}^{\ell} \quad j \in J, \ell \in L, s \in S \\
& \sum_{j \in J} x_{i j s}^{\ell} \leq K_{i}^{\ell} z_{i s}^{\ell} \quad i \in I, \ell \in L, s \in S \\
& \Lambda_{j s}^{\ell} \geq 0 \quad j \in J, \ell \in L, s \in S \\
& y_{i j} \in\{0,1\} \quad i \in I, j \in J
\end{aligned}
$$




$$
\begin{aligned}
& x_{i j s}^{\ell} \geq 0 \quad i \in I, j \in J, \ell \in L, s \in S \\
& z_{i s}^{\ell} \in\{0,1\} \quad i \in I, \ell \in L, s \in S
\end{aligned}
$$

In this formulation, $\Lambda_{j s}^{\ell}$ denotes the amount of commodity $\ell$ requested by customer $j$ which is not supplied if the demand level is $s(\ell \in L, j \in J, s \in S)$.

\subsection{Measuring the relevance of using a stochastic approach}

Although being a well-known fact that no measure exists which is robust for measuring the relevance of using a stochastic programming approach, most of the authors rely on measures which, at least, can give some indication of this relevance. Among these measures, we have considered the value of the stochastic solution (VSS) (the reader can refer to Birge (1982) and Birge and Louveaux (1997) for the use of this measure in twostage stochastic problems and to Escudero et al. (2007) for a multi-stage setting). In addition, to get a better perception on the relevance of the stochastic solution across all scenarios, we considered two additional measures. The first one relates somehow with the VSS. The second measure is related with the expected value of perfect information (EVPI) (see also Birge and Louveaux (1997) for the use of this measure in stochastic programming problems).

To compute the VSS, the random variables are replaced by their expected values and the resulting deterministic problem is solved. The optimal solution to this problem gives a first-stage feasible solution to the original stochastic problem because the original first-stage constraints and variables appear unchanged in this deterministic problem. Furthermore, the fact that the problem has relatively complete recourse assures that such first-stage solution has a feasible completion in terms of the secondstage problem. Thus, it can be considered as a first-stage solution to the stochastic problem. Solving the stochastic problem fixing this first-stage solution leads to a complete solution to the stochastic problem. The difference between the value of this solution-say $\mathrm{AEV}$ - and the optimal value of the stochastic problem-say $\mathrm{SP}$ - is the value of the stochastic solution, i.e., VSS $=\mathrm{AEV}-\mathrm{SP}$.

For the above reasons, it becomes clear that the solution corresponding to AEV can be considered as an approximated solution to the stochastic problem. Furthermore, as we shall see, for the uncapacitated version of the problem, such solution is usually easier to obtain and provides tight gaps.

In our case, replacing the random variables $D_{j}^{\ell}$ by their expectation-say $\bar{D}_{j}^{\ell}$ leads to a deterministic problem that we include below (in its capacitated version) for the sake of completeness.

$$
\begin{array}{ll}
\min & \sum_{i \in I} \sum_{j \in J} c_{i j} y_{i j}+\sum_{i \in I} \sum_{j \in J} \sum_{\ell \in L} d_{i j}^{\ell} x_{i j}^{\ell}+\sum_{i \in I} \sum_{\ell \in L} h_{i}^{\ell} z_{i}^{\ell}+\sum_{j \in J} \sum_{\ell \in L} \psi_{j}^{\ell} \Lambda_{j}^{\ell} \\
\text { s.t. } & \sum_{i \in I} y_{i j} \geq 1 \quad j \in J \\
& \sum_{\ell \in L} x_{i j}^{\ell} \leq \sum_{\ell \in L} \bar{D}_{j}^{\ell} y_{i j} \quad i \in I, j \in J
\end{array}
$$




$$
\begin{aligned}
& \Lambda_{j}^{\ell} \geq \bar{D}_{j}^{\ell}-\sum_{i \in I} x_{i j}^{\ell} \quad j \in J, \ell \in L \\
& \sum_{j \in J} x_{i j}^{\ell} \leq K_{i}^{\ell} z_{i}^{\ell} \quad i \in I, \quad \ell \in L \\
& \Lambda_{j}^{\ell} \geq 0 \quad j \in J, \ell \in L \\
& y_{i j} \in\{0,1\} \quad i \in I, j \in J \\
& x_{i j}^{\ell} \geq 0 \quad i \in I, j \in J, \ell \in L \\
& z_{i}^{\ell} \in\{0,1\} \quad i \in I, \quad \ell \in L
\end{aligned}
$$

Note that model (52)-(60) results from model (43)-(51) by considering a single demand level, which is the one corresponding to the demands levels being defined according to $\bar{D}_{j}^{\ell}$.

A simple way for obtaining $\mathrm{AEV}$ is first to obtain the set of values $\mathrm{EV}_{s}(s \in S)$, each of which representing the optimal value of the problem we can define for each scenario $s \in S$ when the first-stage variables are fixed according to the optimal solution to the deterministic problem. Then, we can simply set $\mathrm{AEV}=\sum_{s \in S} p_{s} \mathrm{EV}_{s}$.

Analogously, the value SP (optimal value of the stochastic problem) can be written as a weighted sum of the set of values $\mathrm{SP}_{s}(s \in S)$, each of which representing the optimal value of the objective function under scenario $s$ when the first-stage variables are fixed to the optimal values obtained in the stochastic version of Problem (43)-(51). In particular, $\mathrm{SP}=\sum_{s \in S} p_{S} S P_{S}$.

Taking the previous aspects into account, we can write

$$
\mathrm{VSS}=\mathrm{AEV}-\mathrm{SP}=\sum_{s \in S} p_{s}\left(\mathrm{EV}_{s}-\mathrm{SP}_{s}\right)
$$

Looking into the previous formula, we realize that we have a weighted deviation, which is dependent on the magnitude of the values involved. To get a better perception of this value, we can consider the relative percent VSS, which can be computed as

$$
\mathrm{VSS}_{R}=100 \times \frac{1}{S P}(\mathrm{~A} E V-\mathrm{S} P)=100 \times \frac{1}{\mathrm{~S} P} \sum_{s \in S} p_{s}\left(\mathrm{E} V_{s}-\mathrm{S} P_{S}\right) .
$$

Although relevant, the previous expression tells nothing about the behavior of the deterministic solution across all scenarios. An alternative we consider is the following modified measure:

$$
\mathrm{EEV}_{R}=100 \times \sum_{s \in S} p_{s} \frac{\mathrm{EV}_{s}-\mathrm{SP}_{s}}{\mathrm{SP}_{S}}
$$

The EVPI represents the maximum amount that the decision maker would be willing to pay in order to get perfect information. This value is computed as the difference between the expected value with (additional) perfect information about the realized 
scenario and the expected value without knowing the actual state of nature. Thus, EVPI measures the value of the stochastic solution as compared to the values of actually realized solutions.

To obtain the EVPI we can start by computing the set of values $\mathrm{WS}_{s}(s \in S)$, each of which representing the optimal value of the problem we can associate with each scenario (i.e., assuming perfect information). These values can be gathered into the well-known wait-and-see solution, which is commonly denoted by WS. Accordingly,

$$
\mathrm{WS}=\sum_{s \in S} p_{s} \mathrm{WS}_{S}
$$

and the EVPI is defined as

$$
\mathrm{EVPI}=\mathrm{SP}-\mathrm{WS}=\sum_{s \in S} p_{s}\left(\mathrm{SP}_{s}-\mathrm{WS}_{s}\right)
$$

Again, this measure says little about the individual scenarios. We consider, as an alternative, a measure which not only is independent from the magnitude of the values involved but also captures some information across all scenarios:

$$
\mathrm{EVPI}_{R}=100 \times \sum_{s \in S} p_{s} \frac{\mathrm{SP}_{s}-\mathrm{WS}_{s}}{\mathrm{WS}_{s}} .
$$

In synthesis, for the problem addressed in this paper, we measure the relevance of using a stochastic approach using $\operatorname{VSS}_{R}, \mathrm{EEV}_{R}$ and $\mathrm{EVPI}_{R}$.

\section{Computational tests}

In this section, we report the results of a series of computational tests performed to evaluate the behavior of the models proposed in Sects. 2 and 3. It is also a goal with these tests to understand the extent to which a commercial solver can be used to solve the addressed problems to optimality.

All computational tests have been performed on a PC with a Intel(R) Core(TM) i7 processor with $2.80 \mathrm{GHz}$ and $8 \mathrm{~GB}$ of RAM. Programs were implemented using Visual C++ 2010 Express and ILOG CPLEX Studio Academic 12.5.1 with the Concert Technology routines. Default parameters have been used.

\subsection{Test data}

Before generating the instances, we identified four relevant factors: the number of (i) origins, (ii) destinations, (iii) scenarios and (iv) commodities. For each of these factors, we consider different possibilities which determine the dimensions of the test instances. In particular, $|I|$ is chosen in the set $\{10,20,50\},|J|$ in $\{20,50,100\},|S|$ in $\{8,12,20,30\}$ and $|L|$ in $\{1,2,3\}$. 
Once the dimensions are set, one instance was generated as follows:

- The unitary flow shipment costs $d_{i j}^{l}$ were drawn from a uniform distribution $U[10,100]$.

- The fixed handling costs $h_{i}^{l}$ were drawn from a uniform distribution $U[500,3500]$.

- The set-up costs $c_{i j}(i \in I, j \in J)$ were drawn from a uniform distribution centered at $\frac{55 \times \mathrm{D} T}{|I| \times|J|}$ with range $\pm \frac{1}{4} \times \frac{55 \times \mathrm{D} T}{|I| \times|J|}$, where $\mathrm{D} T=\frac{680}{3} \times|J| \times|L|$ is a reference value for the total demand level. Note that DT is not an estimate of the total demand level for one scenario. Furthermore, this estimation is considered only for generating the distribution of the set-up cost for the links, i.e., the costs associated with the network design. We recall that the design of the network is to be decided before the actual demand is disclosed, which means that the network design chosen will be used no matter the scenario that will actually occur. Accordingly, the value DT was computed assuming equal probability for every scenario.

- Demand ranges were generated according to a uniform distribution. Three possible intervals were considered: (i) $U[10,50]$, (ii) $U[100,200]$ and (iii) $U[400,600]$.

- Our data sets always include the following four different types of scenarios: (S1) all the demand levels are drawn from interval (i); (S2) all the demand levels are drawn from interval (ii); (S3) all the demand levels are drawn from interval (iii) and; (S4) For each customer a demand level is initially chosen randomly considering the same probability for the three different intervals. Several scenarios of each type were considered depending on the total number of demand levels to be drawn ( 8 , 12, 20 or 30). They are summarized below:

\begin{tabular}{llllc}
\hline$|S|$ & $\left|S_{1}\right|$ & $\left|S_{2}\right|$ & $\left|S_{3}\right|$ & $\left|S_{4}\right|$ \\
\hline 8 & 1 & 1 & 2 & 4 \\
12 & 1 & 1 & 2 & 8 \\
20 & 1 & 1 & 4 & 12 \\
30 & 3 & 3 & 6 & 18 \\
\hline
\end{tabular}

- $p_{s}=P[\xi=s]=\frac{1}{|S|}$.

- The maximum amount of commodity $\ell$ available at origin $i, K_{i}^{\ell}$ is drawn from a uniform distribution centered at $\frac{\mathrm{D} T}{|I| \times|L|}$ with range $\pm 0.2 \times \frac{\mathrm{D} T}{|I| \times|L|}$.

- The unitary penalty costs for not supplying the demand of customer $j$ in terms of commodity $\ell, \psi_{j}^{\ell}$, was drawn from a uniform distribution centered at $\beta=$ $100+\frac{3500+\alpha+\alpha / 4}{680 / 3}$ with range $\pm 0.1 \times \beta$, where $\alpha=\frac{55 \times \mathrm{DT}}{|I| \times|J|}$.

For each combination of $|I|,|J|,|L|$ and $|S|$, five instances were generated for the uncapacitated case as well as for the capacitated one. In total, approximately 1,000 instances were generated.

Remark 3 1. The cardinalities considered for $|I|,|J|,|S|$ and $|L|$ define the number of binary and continuous variables to be used. In Table 1, we find this information for stochastic capacitated multi-commodity problem (43)-(51). For each combination 
Table 1 Dimension of the test instances for the stochastic capacitated multi-commodity problem (43)-(51).

\begin{tabular}{|c|c|c|c|c|c|c|c|c|}
\hline \multirow[t]{2}{*}{$|I|$} & \multirow[t]{2}{*}{$|J|$} & \multirow[t]{2}{*}{$|S|$} & \multicolumn{2}{|l|}{$|L|=1$} & \multicolumn{2}{|l|}{$|L|=2$} & \multicolumn{2}{|l|}{$|L|=3$} \\
\hline & & & Binary & Continuous & Binary & Continuous & Binary & Continuous \\
\hline \multirow[t]{15}{*}{10} & \multirow[t]{5}{*}{20} & 1 & 210 & 220 & 220 & 440 & 230 & 660 \\
\hline & & 8 & 280 & 1,760 & 360 & 3,520 & 440 & 5,280 \\
\hline & & 12 & 320 & 2,640 & 440 & 5,280 & 560 & 7,920 \\
\hline & & 20 & 400 & 4,400 & 600 & 8,800 & 800 & 13,200 \\
\hline & & 30 & 500 & 6,600 & 800 & 13,200 & 1,100 & 19,800 \\
\hline & \multirow[t]{5}{*}{50} & 1 & 510 & 550 & 520 & 1,100 & 530 & 1,650 \\
\hline & & 8 & 580 & 4,400 & 660 & 8,800 & 740 & 13,200 \\
\hline & & 12 & 620 & 6,600 & 740 & 13,200 & 860 & 19,800 \\
\hline & & 20 & 700 & 11,000 & 900 & 22,000 & 1,100 & 33,000 \\
\hline & & 30 & 800 & 16,500 & 1,100 & 33,000 & 1,400 & 49,500 \\
\hline & \multirow[t]{5}{*}{100} & 1 & 1,010 & 1,100 & 1,020 & 2,200 & 1,030 & 3,300 \\
\hline & & 8 & 1,080 & 8,800 & 1,160 & 17,600 & 1,240 & 26,400 \\
\hline & & 12 & 1,120 & 13,200 & 1,240 & 26,400 & 1,360 & 39,600 \\
\hline & & 20 & 1,200 & 22,000 & 1,400 & 44,000 & 1,600 & 66,000 \\
\hline & & 30 & 1,300 & 33,000 & 1,600 & 66,000 & 1,900 & 99,000 \\
\hline \multirow[t]{15}{*}{20} & \multirow[t]{5}{*}{20} & 1 & 420 & 420 & 440 & 840 & 460 & 1,260 \\
\hline & & 8 & 560 & 3,360 & 720 & 6,720 & 880 & 10,080 \\
\hline & & 12 & 640 & 5,040 & 880 & 10,080 & 1,120 & 15,120 \\
\hline & & 20 & 800 & 8,400 & 1,200 & 16,800 & 1,600 & 25,200 \\
\hline & & 30 & 1,000 & 12,600 & 1,600 & 25,200 & 2,200 & 37,800 \\
\hline & \multirow[t]{5}{*}{50} & 1 & 1,020 & 1,050 & 1,040 & 2,100 & 1,060 & 3,150 \\
\hline & & 8 & 1,160 & 8,400 & 1,320 & 16,800 & 1,480 & 25,200 \\
\hline & & 12 & 1,240 & 12,600 & 1,480 & 25,200 & 1,720 & 37,800 \\
\hline & & 20 & 1,400 & 21,000 & 1,800 & 42,000 & 2,200 & 63,000 \\
\hline & & 30 & 1,600 & 31,500 & 22,00 & 63,000 & 2,800 & 94,500 \\
\hline & \multirow[t]{5}{*}{100} & 1 & 2,020 & 2,100 & 2,040 & 4,200 & 2,060 & 6,300 \\
\hline & & 8 & 2,160 & 16,800 & 2,320 & 33,600 & 2,480 & 50,400 \\
\hline & & 12 & 2,240 & 25,200 & 2,480 & 50,400 & 2,720 & 75,600 \\
\hline & & 20 & 2,400 & 42,000 & 2,800 & 84,000 & 3,200 & 126,000 \\
\hline & & 30 & 2,600 & 63,000 & 3,200 & 126,000 & 3,800 & 189,000 \\
\hline \multirow[t]{10}{*}{50} & \multirow[t]{5}{*}{50} & 1 & 2,550 & 2,550 & 2,600 & 5,100 & 2,650 & 7,650 \\
\hline & & 8 & 2,900 & 20,400 & 3,300 & 40,800 & 3,700 & 6,1200 \\
\hline & & 12 & 3,100 & 30,600 & 3,700 & 61,200 & 4,300 & 91,800 \\
\hline & & 20 & 3,500 & 51,000 & 4,500 & 102,000 & 5,500 & 153,000 \\
\hline & & 30 & 4,000 & 76,500 & 5,500 & 153,000 & 7,000 & 229,500 \\
\hline & \multirow[t]{5}{*}{100} & 1 & 5,050 & 5,100 & 5,100 & 10,200 & 5,150 & 15,300 \\
\hline & & 8 & 5,400 & 40,800 & 5,800 & 81,600 & 6,200 & 122,400 \\
\hline & & 12 & 5,600 & 61,200 & 6,200 & 122,400 & 6,800 & 183,600 \\
\hline & & 20 & 6,000 & 102,000 & 7,000 & 204,000 & 8,000 & 306,000 \\
\hline & & 30 & 6,500 & 153,000 & 8,000 & 306,000 & 9,500 & 459,000 \\
\hline
\end{tabular}


of $|I|,|J|$ and $|L|$, we include a line for $|S|=1$. This line corresponds to the dimension of the expected value problem (a single scenario which is defined by the average demand levels). Observing this table, we realize that some instances are quite large namely in terms of the number of binary variables. These dimensions are relevant even considering the state-of-the-art in terms of mixed-integer linear stochastic programming. For instance, considering a general two-stage mixedinteger linear stochastic problem, Escudero et al. (2012) tackle test instances which, for a compact representation (as we consider in this paper), have up to 20 binary variables in the first stage and 6,300 in the second stage.

2. A scenario is a complete realization of the joint random vector of demand levels. Nevertheless, the recourse function is based on the choice of the network design. This way, in our model, the combinatorial choice of the network design is put together with all the possible scenarios that may occur, which leads to a huge number of possibilities.

3. Despite the comment above, at a first glance, one might think that the number of scenarios is too small. However, it should be noted that in practice, uncertainty in the demand level for some product or service is captured via a finite number of scenarios or using some probability law. In the first case, one possibility is to consider a base demand level (estimated, for instance, using some marketing research approach) and some variations from this level (e.g., $20 \%$ above and below). This means that in total, the number of scenarios is not large. In fact, in such a case, it would be difficult to sustain 100 or 500 scenarios drawn from a simple estimate. In the second case, when a probability law can be adjusted to the demand level, the number of scenarios is typically infinity (apart from some exceptions as it is the case with the binomial distribution which, nevertheless, is not often found in the literature for describing demand). With an infinite number of scenarios, the models proposed in this paper (like the large majority of the stochastic programming models published in the literature) cannot be used because they cannot be solved explicitly. Accordingly, one has to resort to some sampling method (e.g., sample average approximation) which relies on a set of samples (of scenarios) drawn from the underlying distribution. In this case, a sequence of finite-scenario problems has to be solved. For convergence purposes, the number of scenarios does not need to be high as far as more problems can be solved.

4. It is important to note once more that in our modeling framework we are considering at the same time the design of the network and the stochasticity of the demand. This way, to see how the design is affected by the demand, we have to consider, apart from homogenous cases, also extreme cases. This explains the choices made for demand level generation. We also recall that as observed before, for homogenous cases, the solutions of our model will tend to behave close to the the solution of an uncapacitated facility location problem.

\subsection{Computational results}

As mentioned above, we have used the solver ILOG CPLEX Studio Academic 12.5.1 to solve the generated instances. In particular, we registered the cpu time (in seconds) 
Table 2 Average gaps and CPU time (in seconds) for the stochastic uncapacitated multi-commodity problem

\begin{tabular}{|c|c|c|c|c|c|c|c|c|c|c|c|c|c|c|}
\hline \multirow[t]{2}{*}{$|I|$} & \multirow[t]{2}{*}{$|J|$} & \multirow[t]{2}{*}{$|S|$} & \multicolumn{4}{|c|}{$|L|=1$} & \multicolumn{4}{|c|}{$|L|=2$} & \multicolumn{4}{|c|}{$|L|=3$} \\
\hline & & & Gap & $\mathrm{CPU}$ & CPUmax & Opt & Gap & $\mathrm{CPU}$ & CPUmax & Opt & Gap & $\mathrm{CPU}$ & CPUmax & Opt \\
\hline \multirow[t]{12}{*}{10} & \multirow[t]{4}{*}{20} & 8 & 0.00 & 0.20 & 1.00 & 5 & 0.00 & 0.60 & 1.00 & 5 & 0.00 & 0.80 & 1.00 & 5 \\
\hline & & 12 & 0.00 & 0.20 & 1.00 & 5 & 0.00 & 1.00 & 1.00 & 5 & 0.00 & 2.80 & 4.00 & 5 \\
\hline & & 20 & 0.00 & 0.20 & 1.00 & 5 & 0.00 & 1.80 & 3.00 & 5 & 0.00 & 5.40 & 7.00 & 5 \\
\hline & & 30 & 0.00 & 0.40 & 1.00 & 5 & 0.00 & 3.00 & 4.00 & 5 & 0.00 & 19.80 & 25.00 & 5 \\
\hline & \multirow[t]{4}{*}{50} & 8 & 0.00 & 0.40 & 1.00 & 5 & 0.00 & 0.80 & 2.00 & 5 & 0.00 & 2.00 & 3.00 & 5 \\
\hline & & 12 & 0.00 & 0.40 & 1.00 & 5 & 0.00 & 1.40 & 2.00 & 5 & 0.00 & 4.20 & 5.00 & 5 \\
\hline & & 20 & 0.00 & 0.60 & 1.00 & 5 & 0.00 & 2.80 & 3.00 & 5 & 0.00 & 6.40 & 8.00 & 5 \\
\hline & & 30 & 0.00 & 1.00 & 1.00 & 5 & 0.00 & 4.20 & 6.00 & 5 & 0.00 & 25.60 & 37.00 & 5 \\
\hline & \multirow[t]{4}{*}{100} & 8 & 0.00 & 0.60 & 1.00 & 5 & 0.00 & 2.00 & 2.00 & 5 & 0.00 & 5.20 & 7.00 & 5 \\
\hline & & 12 & 0.00 & 0.60 & 1.00 & 5 & 0.00 & 3.00 & 4.00 & 5 & 0.00 & 7.20 & 9.00 & 5 \\
\hline & & 20 & 0.00 & 1.40 & 2.00 & 5 & 0.00 & 4.60 & 5.00 & 5 & 0.00 & 13.20 & 17.00 & 5 \\
\hline & & 30 & 0.00 & 2.40 & 3.00 & 5 & 0.00 & 7.80 & 9.00 & 5 & 0.00 & 23.40 & 31.00 & 5 \\
\hline \multirow[t]{12}{*}{20} & \multirow[t]{4}{*}{20} & 8 & 0.00 & 0.20 & 1.00 & 5 & 0.00 & 1.60 & 2.00 & 5 & 0.00 & 6.20 & 9.00 & 5 \\
\hline & & 12 & 0.00 & 0.60 & 2.00 & 5 & 0.00 & 3.60 & 5.00 & 5 & 0.00 & 16.40 & 23.00 & 5 \\
\hline & & 20 & 0.00 & 1.00 & 2.00 & 5 & 0.00 & 8.80 & 11.00 & 5 & 0.00 & 29.80 & 47.00 & 5 \\
\hline & & 30 & 0.00 & 0.80 & 1.00 & 5 & 0.00 & 23.20 & 37.00 & 5 & 0.00 & 60.20 & 76.00 & 5 \\
\hline & \multirow[t]{4}{*}{50} & 8 & 0.00 & 1.00 & 1.00 & 5 & 0.00 & 5.00 & 6.00 & 5 & 0.00 & 20.40 & 23.00 & 5 \\
\hline & & 12 & 0.00 & 1.20 & 2.00 & 5 & 0.00 & 11.00 & 18.00 & 5 & 0.00 & 27.00 & 38.00 & 5 \\
\hline & & 20 & 0.00 & 1.20 & 2.00 & 5 & 0.00 & 12.40 & 20.00 & 5 & 0.00 & 62.20 & 75.00 & 5 \\
\hline & & 30 & 0.00 & 2.60 & 4.00 & 5 & 0.00 & 44.00 & 55.00 & 5 & 0.00 & 203.60 & 279.00 & 5 \\
\hline & \multirow[t]{4}{*}{100} & 8 & 0.00 & 1.20 & 2.00 & 5 & 0.00 & 3.20 & 4.00 & 5 & 0.00 & 10.60 & 12.00 & 5 \\
\hline & & 12 & 0.00 & 1.60 & 3.00 & 5 & 0.00 & 6.40 & 7.00 & 5 & 0.00 & 29.60 & 43.00 & 5 \\
\hline & & 20 & 0.00 & 2.80 & 3.00 & 5 & 0.00 & 11.00 & 18.00 & 5 & 0.00 & 57.00 & 65.00 & 5 \\
\hline & & 30 & 0.00 & 3.80 & 4.00 & 5 & 0.00 & 33.20 & 46.00 & 5 & 0.00 & 205.40 & 257.00 & 5 \\
\hline \multirow[t]{8}{*}{50} & \multirow[t]{4}{*}{50} & 8 & 0.00 & 6.80 & 10.00 & 5 & 0.00 & 46.20 & 93.00 & 5 & 0.00 & 157.80 & 196.00 & 5 \\
\hline & & 12 & 0.00 & 11.00 & 15.00 & 5 & 0.00 & 182.20 & 481.00 & 5 & 0.00 & 954.40 & $2,350.00$ & 5 \\
\hline & & 20 & 0.00 & 23.60 & 48.00 & 5 & 0.00 & 218.80 & 293.00 & 5 & 0.00 & $2,204.80$ & $3,165.00$ & 5 \\
\hline & & 30 & 0.00 & 54.20 & 112.00 & 5 & 0.00 & $1,739.80$ & $3,365.00$ & 5 & 0.00 & $8,108.00$ & $14,625.00$ & 5 \\
\hline & \multirow[t]{4}{*}{100} & 8 & 0.00 & 13.20 & 18.00 & 5 & 0.00 & 153.00 & 280.00 & 5 & 0.00 & $1,751.60$ & $5,558.00$ & 5 \\
\hline & & 12 & 0.00 & 20.60 & 24.00 & 5 & 0.00 & 422.00 & 756.00 & 5 & 0.00 & $6,764.00$ & $19,374.00$ & 5 \\
\hline & & 20 & 0.00 & 51.00 & 74.00 & 5 & 0.00 & $1,008.20$ & $1,565.00$ & 5 & 0.01 & $10,789.20$ & $36,003.00$ & 4 \\
\hline & & 30 & 0.00 & 210.00 & 546.00 & 5 & 0.01 & $7,978.75$ & $36,002.00$ & 4 & 0.06 & - & - & 0 \\
\hline
\end{tabular}

required to obtain an optimal solution for each instance. A time limit of 36,000 s (10 h) was considered. For those instances where the time limit was reached, the relative gap between the objective value of the best integer solution and the best-known lower bound on the optimal solution value was saved.

Tables 2, 3, 4, 5 and 6 report the results obtained for the instances associated with each combination of the parameters $|I|,|J|,|L|$ and $|S|$. In all tables, we can find the rows grouped into several blocks, one for each value of $|I|$. Within each block, a row 
Table 3 Average gaps and CPU time (in seconds) for the stochastic capacitated multi-commodity problem

\begin{tabular}{|c|c|c|c|c|c|c|c|c|c|c|c|}
\hline \multirow[t]{2}{*}{$|I|$} & \multirow[t]{2}{*}{$|J|$} & \multirow[t]{2}{*}{$|S|$} & \multicolumn{3}{|c|}{$|L|=1$} & \multicolumn{3}{|c|}{$|L|=2$} & \multicolumn{3}{|c|}{$|L|=3$} \\
\hline & & & Gap & CPU & Opt & Gap & $\mathrm{CPU}$ & Opt & Gap & CPU & Opt \\
\hline \multirow[t]{12}{*}{10} & \multirow[t]{4}{*}{20} & 8 & 0.00 & 42.33 & 5 & 0.00 & $5,333.67$ & 5 & 0.40 & $35,520.67$ & 1 \\
\hline & & 12 & 0.00 & $1,335.67$ & 5 & 0.43 & $27,849.33$ & 1 & 1.83 & - & 0 \\
\hline & & 20 & 0.00 & $5,307.67$ & 5 & 1.24 & - & 0 & 2.09 & - & 0 \\
\hline & & 30 & 0.49 & - & 0 & 2.34 & - & 0 & 2.51 & - & 0 \\
\hline & \multirow[t]{4}{*}{50} & 8 & 0.00 & $1,164.33$ & 5 & 0.26 & - & 0 & 0.39 & - & 0 \\
\hline & & 12 & 0.28 & - & 0 & 0.68 & - & 0 & 0.78 & - & 0 \\
\hline & & 20 & 0.53 & - & 0 & 0.83 & - & 0 & 0.97 & - & 0 \\
\hline & & 30 & 0.80 & - & 0 & 1.05 & - & 0 & 1.15 & - & 0 \\
\hline & \multirow[t]{4}{*}{100} & 8 & 0.05 & - & 0 & 0.12 & - & 0 & 0.15 & - & 0 \\
\hline & & 12 & 0.25 & - & 0 & 0.31 & - & 0 & 0.37 & - & 0 \\
\hline & & 20 & 0.40 & - & 0 & 0.32 & - & 0 & 0.40 & - & 0 \\
\hline & & 30 & 0.45 & - & 0 & 0.53 & - & 0 & 0.61 & - & 0 \\
\hline \multirow[t]{12}{*}{20} & \multirow[t]{4}{*}{20} & 8 & 0.05 & $12,699.33$ & 2 & 0.65 & - & 0 & 0.92 & - & 0 \\
\hline & & 12 & 0.20 & $33,927.67$ & 1 & 0.98 & - & 0 & 1.54 & - & 0 \\
\hline & & 20 & 0.40 & - & 0 & 1.17 & - & 0 & 1.73 & - & 0 \\
\hline & & 30 & 0.87 & - & 0 & 1.59 & - & 0 & 2.36 & - & 0 \\
\hline & \multirow[t]{4}{*}{50} & 8 & 0.35 & - & 0 & 1.10 & - & 0 & 1.43 & - & 0 \\
\hline & & 12 & 0.87 & - & 0 & 2.16 & - & 0 & 2.46 & - & 0 \\
\hline & & 20 & 1.02 & - & 0 & 2.14 & - & 0 & 2.84 & - & 0 \\
\hline & & 30 & 1.66 & - & 0 & 2.85 & - & 0 & 4.04 & - & 0 \\
\hline & \multirow[t]{4}{*}{100} & 8 & 0.30 & - & 0 & 0.50 & - & 0 & 0.76 & - & 0 \\
\hline & & 12 & 0.62 & - & 0 & 1.20 & - & 0 & 1.40 & - & 0 \\
\hline & & 20 & 0.66 & - & 0 & 0.99 & - & 0 & 1.65 & - & 0 \\
\hline & & 30 & 1.06 & - & 0 & 1.82 & - & 0 & 1.88 & - & 0 \\
\hline \multirow[t]{8}{*}{50} & \multirow[t]{4}{*}{50} & 8 & 0.40 & - & 0 & 0.97 & - & 0 & 1.32 & - & 0 \\
\hline & & 12 & 0.65 & - & 0 & 1.39 & - & 0 & 2.54 & - & 0 \\
\hline & & 20 & 0.72 & - & 0 & 1.86 & - & 0 & 2.30 & - & 0 \\
\hline & & 30 & 1.16 & - & 0 & 2.95 & - & 0 & 10.40 & - & 0 \\
\hline & \multirow[t]{4}{*}{100} & 8 & 0.48 & - & 0 & 1.35 & - & 0 & 2.50 & - & 0 \\
\hline & & 12 & 1.16 & - & 0 & 3.00 & - & 0 & 4.13 & - & 0 \\
\hline & & 20 & 1.58 & - & 0 & 2.79 & - & 0 & 10.15 & - & 0 \\
\hline & & 30 & 2.06 & - & 0 & 8.78 & - & 0 & 13.78 & - & 0 \\
\hline
\end{tabular}

corresponds to a set of instances with a fixed number of destinations $|J|$ and demand levels $|S|$. Furthermore, the columns are also grouped into blocks, one for each value of $|L|$. Thus, each entry in these tables corresponds to a value computed over all the instances associated with a particular combination of $|I|,|J|,|L|$ and $|S|$.

In Tables 2 and 3, we can observe results for the stochastic uncapacitated and capacitated problems, respectively. Each entry in the columns headed by 'Gap' con- 
Table 4 Percentage relative VSS and the corresponding CPU time (in seconds) for the uncapacitated multi-commodity problem

\begin{tabular}{|c|c|c|c|c|c|c|c|c|}
\hline \multirow[t]{2}{*}{$|I|$} & \multirow[t]{2}{*}{$|J|$} & \multirow[t]{2}{*}{$|S|$} & \multicolumn{2}{|l|}{$|L|=1$} & \multicolumn{2}{|l|}{$|L|=2$} & \multicolumn{2}{|l|}{$|L|=3$} \\
\hline & & & $V S S_{R}$ & CPU & $V S S_{R}$ & $\mathrm{CPU}$ & $V S S_{R}$ & $\mathrm{CPU}$ \\
\hline \multirow[t]{12}{*}{10} & \multirow[t]{4}{*}{20} & 8 & 0.02 & 0.00 & 0.00 & 0.40 & 0.00 & 0.80 \\
\hline & & 12 & 0.00 & 0.20 & 0.00 & 0.40 & 0.03 & 0.80 \\
\hline & & 20 & 0.00 & 0.20 & 0.00 & 0.40 & 0.04 & 1.00 \\
\hline & & 30 & 0.00 & 0.20 & 0.05 & 0.80 & 0.06 & 1.00 \\
\hline & \multirow[t]{4}{*}{50} & 8 & 0.00 & 0.20 & 0.00 & 0.60 & 0.00 & 0.60 \\
\hline & & 12 & 0.00 & 0.20 & 0.00 & 0.00 & 0.00 & 0.80 \\
\hline & & 20 & 0.00 & 0.00 & 0.00 & 0.40 & 0.00 & 1.00 \\
\hline & & 30 & 0.00 & 0.00 & 0.00 & 0.40 & 0.00 & 1.00 \\
\hline & \multirow[t]{4}{*}{100} & 8 & 0.00 & 0.20 & 0.00 & 0.40 & 0.00 & 0.60 \\
\hline & & 12 & 0.00 & 0.20 & 0.00 & 0.40 & 0.00 & 1.20 \\
\hline & & 20 & 0.00 & 0.40 & 0.00 & 0.20 & 0.00 & 1.20 \\
\hline & & 30 & 0.00 & 0.20 & 0.00 & 0.40 & 0.00 & 1.20 \\
\hline \multirow[t]{12}{*}{20} & \multirow[t]{4}{*}{20} & 8 & 0.00 & 0.20 & 0.06 & 0.80 & 0.06 & 0.80 \\
\hline & & 12 & 0.03 & 0.20 & 0.07 & 0.80 & 0.08 & 1.00 \\
\hline & & 20 & 0.07 & 0.40 & 0.07 & 1.20 & 0.14 & 1.40 \\
\hline & & 30 & 0.13 & 0.20 & 0.15 & 0.80 & 0.23 & 1.40 \\
\hline & \multirow[t]{4}{*}{50} & 8 & 0.01 & 0.20 & 0.03 & 0.40 & 0.04 & 1.20 \\
\hline & & 12 & 0.01 & 0.40 & 0.05 & 0.00 & 0.01 & 1.00 \\
\hline & & 20 & 0.01 & 0.20 & 0.03 & 0.60 & 0.03 & 1.40 \\
\hline & & 30 & 0.03 & 0.40 & 0.02 & 1.00 & 0.04 & 2.00 \\
\hline & \multirow[t]{4}{*}{100} & 8 & 0.00 & 0.20 & 0.00 & 0.60 & 0.00 & 1.40 \\
\hline & & 12 & 0.00 & 0.40 & 0.00 & 1.00 & 0.01 & 1.60 \\
\hline & & 20 & 0.00 & 0.00 & 0.00 & 1.20 & 0.00 & 1.20 \\
\hline & & 30 & 0.00 & 0.20 & 0.00 & 1.40 & 0.01 & 2.60 \\
\hline \multirow[t]{8}{*}{50} & \multirow[t]{4}{*}{50} & 8 & 0.29 & 1.40 & 0.07 & 1.00 & 0.07 & 1.60 \\
\hline & & 12 & 0.22 & 1.40 & 0.03 & 1.60 & 0.05 & 2.40 \\
\hline & & 20 & 0.22 & 1.20 & 0.03 & 1.40 & 0.04 & 2.40 \\
\hline & & 30 & 0.37 & 1.40 & 0.04 & 2.20 & 0.10 & 3.20 \\
\hline & \multirow[t]{4}{*}{100} & 8 & 0.10 & 0.60 & 0.01 & 2.00 & 0.02 & 4.60 \\
\hline & & 12 & 0.08 & 0.20 & 0.03 & 3.40 & 0.02 & 5.20 \\
\hline & & 20 & 0.08 & 0.00 & 0.03 & 2.80 & 0.01 & 5.80 \\
\hline & & 30 & 0.14 & 0.80 & 0.02 & 4.00 & 0.04 & 8.00 \\
\hline
\end{tabular}

tains an average of percentage gaps. Each gap is defined by $100 \times$ (bestinteger bestobjective)/bestobjective, where bestinteger is the objective value of the best integer solution and bestobjective is the best-known lower bound on the optimal solution found by the solver. An entry in the columns headed by 'CPU' gives the average cpu time (seconds) required by the solver to obtain the optimal/best solution. An entry in the columns headed by 'CPUmax' gives the maximum cpu time (seconds) among the instances corresponding to the entry. In addition, an entry in the columns headed by 'Opt' indicates the number of instances that could be solved to optimality within the time limit. Finally, the symbol "-" in some entry indicates that the time limit was reached for all the five instances corresponding to the entry.

Observing Tables 2 and 3, we can immediately realize that there is a clear difference between the uncapacitated and the capacitated cases. In fact, the inclusion of capacity constraints significantly worsens the efficiency of the solver and consequently, the 
Table 5 Percentage relative VSS and the corresponding CPU time (in seconds) for the capacitated multicommodity problem

\begin{tabular}{|c|c|c|c|c|c|c|c|c|}
\hline \multirow[t]{2}{*}{$|I|$} & \multirow[t]{2}{*}{$|J|$} & \multirow[t]{2}{*}{$|S|$} & \multicolumn{2}{|l|}{$|L|=1$} & \multicolumn{2}{|c|}{$|L|=2$} & \multicolumn{2}{|l|}{$|L|=3$} \\
\hline & & & $V S S_{R}$ & $\mathrm{CPU}$ & $V S S_{R}$ & $\mathrm{CPU}$ & $V S S_{R}$ & CPU \\
\hline \multirow[t]{12}{*}{10} & \multirow[t]{4}{*}{20} & 8 & 9.38 & 1.00 & 5.62 & 6.00 & 4.22 & 25.67 \\
\hline & & 12 & 8.02 & 1.33 & 4.64 & 30.00 & 2.77 & 101.00 \\
\hline & & 20 & 12.20 & 1.33 & 5.58 & 2.67 & 3.70 & 35.33 \\
\hline & & 30 & 5.61 & 2.00 & 3.00 & 43.67 & 1.75 & 88.00 \\
\hline & \multirow[t]{4}{*}{50} & 8 & 9.45 & 3.67 & 4.74 & 24.00 & 4.17 & 705.00 \\
\hline & & 12 & 10.19 & 4.00 & 3.98 & 239.67 & 2.04 & $5,827.33$ \\
\hline & & 20 & 13.08 & 3.67 & 5.67 & 216.00 & 3.49 & 797.00 \\
\hline & & 30 & 5.68 & 31.33 & 1.78 & $3,319.67$ & 1.17 & $1,099.67$ \\
\hline & \multirow[t]{4}{*}{100} & 8 & 10.90 & 25.67 & 4.80 & $12,457.67$ & 2.90 & $10,448.00$ \\
\hline & & 12 & 8.41 & 175.67 & 3.01 & $1,552.33$ & 1.92 & $12,099.33$ \\
\hline & & 20 & 12.02 & 22.33 & 5.85 & $4,326.33$ & 2.61 & $6,990.00$ \\
\hline & & 30 & 4.21 & 96.67 & 1.57 & $4,998.00$ & 1.15 & $4,065.00$ \\
\hline \multirow[t]{12}{*}{20} & \multirow[t]{4}{*}{20} & 8 & 13.94 & 2.33 & 7.88 & 36.67 & 6.28 & $4,915.67$ \\
\hline & & 12 & 15.01 & 20.67 & 8.85 & $1,489.00$ & 7.20 & - \\
\hline & & 20 & 19.53 & 5.67 & 11.69 & 27.67 & 7.40 & 360.33 \\
\hline & & 30 & 13.31 & 9.00 & 7.47 & 815.33 & 4.11 & - \\
\hline & \multirow[t]{4}{*}{50} & 8 & 14.63 & 22.33 & 9.08 & $6,770.67$ & 5.80 & - \\
\hline & & 12 & 13.91 & $1,114.00$ & 7.29 & - & 4.88 & - \\
\hline & & 20 & 20.05 & 30.33 & 10.08 & $9,897.33$ & 5.91 & - \\
\hline & & 30 & 12.09 & $3,010.00$ & 4.45 & - & 1.79 & - \\
\hline & \multirow[t]{4}{*}{100} & 8 & 15.93 & $1,861.33$ & 8.37 & $9,612.00$ & 4.51 & 75.67 \\
\hline & & 12 & 14.73 & $9,605.33$ & 6.63 & - & 3.83 & $2,747.00$ \\
\hline & & 20 & 21.48 & 1.00 & 10.26 & 4.00 & 6.70 & 103.33 \\
\hline & & 30 & 10.12 & 4.33 & 2.97 & - & 1.66 & $4,908.00$ \\
\hline \multirow[t]{8}{*}{50} & \multirow[t]{4}{*}{50} & 8 & 22.30 & $4,899.33$ & 14.47 & - & 9.95 & - \\
\hline & & 12 & 28.03 & - & 17.28 & - & 11.97 & - \\
\hline & & 20 & 31.59 & $6,474.67$ & 19.62 & - & 13.33 & - \\
\hline & & 30 & 22.66 & - & 13.07 & - & 1.30 & - \\
\hline & \multirow[t]{4}{*}{100} & 8 & 21.89 & - & 11.48 & - & 7.80 & - \\
\hline & & 12 & 24.46 & - & 13.91 & - & 7.96 & - \\
\hline & & 20 & 29.97 & - & 16.10 & - & 3.91 & - \\
\hline & & 30 & 18.44 & - & 3.21 & - & -4.66 & - \\
\hline
\end{tabular}

quality of the results. This is not unexpected. In fact, as in many other optimization problems, the inclusion of capacity constraints seems to add extra complexity to the structure of the problem.

Observing in more detail the results in these tables we realize that in the uncapacitated case, we were able solve to optimality all instances even for a large number of origins, destinations and commodities, with the single exception being the set of 
Table 6 Values of $\mathrm{EVPI}_{R}$ and $\mathrm{EEV}_{R}$ for the capacitated multi-commodity problem

\begin{tabular}{|c|c|c|c|c|c|c|c|c|}
\hline \multirow[t]{2}{*}{$|I|$} & \multirow[t]{2}{*}{$|J|$} & \multirow[t]{2}{*}{$|S|$} & \multicolumn{2}{|l|}{$|L|=1$} & \multicolumn{2}{|l|}{$|L|=2$} & \multicolumn{2}{|l|}{$|L|=3$} \\
\hline & & & $\mathrm{EVPI}_{R}$ & $\mathrm{EEV}_{R}$ & $\mathrm{EVPI}_{R}$ & $\mathrm{EEV}_{R}$ & $\mathrm{EVPI}_{R}$ & $\mathrm{EEV}_{R}$ \\
\hline \multirow[t]{12}{*}{10} & \multirow[t]{4}{*}{20} & 8 & 13.68 & 16.69 & 14.63 & 9.23 & 14.94 & 6.76 \\
\hline & & 12 & 14.73 & 11.56 & 14.10 & 6.53 & 15.00 & 3.66 \\
\hline & & 20 & 14.69 & 19.75 & 15.89 & 9.26 & 15.06 & 5.94 \\
\hline & & 30 & 16.15 & 7.79 & 16.92 & 4.23 & 16.31 & 2.27 \\
\hline & \multirow[t]{4}{*}{50} & 8 & 6.83 & 21.63 & 11.17 & 9.73 & 11.61 & 8.69 \\
\hline & & 12 & 7.98 & 17.12 & 11.50 & 6.08 & 12.03 & 3.07 \\
\hline & & 20 & 9.97 & 24.85 & 12.24 & 10.29 & 12.39 & 6.31 \\
\hline & & 30 & 10.45 & 9.00 & 12.44 & 2.42 & 12.23 & 1.61 \\
\hline & \multirow[t]{4}{*}{100} & 8 & 4.21 & 26.77 & 8.49 & 10.59 & 9.32 & 6.46 \\
\hline & & 12 & 5.27 & 14.68 & 8.56 & 4.85 & 10.40 & 2.99 \\
\hline & & 20 & 6.46 & 24.19 & 9.52 & 11.24 & 10.23 & 5.00 \\
\hline & & 30 & 6.98 & 7.32 & 9.81 & 2.33 & 10.75 & 1.34 \\
\hline \multirow[t]{12}{*}{20} & \multirow[t]{4}{*}{20} & 8 & 17.84 & 26.24 & 20.58 & 13.24 & 19.19 & 9.78 \\
\hline & & 12 & 16.46 & 23.52 & 18.64 & 12.38 & 18.41 & 9.52 \\
\hline & & 20 & 16.58 & 33.00 & 18.44 & 17.84 & 17.50 & 11.28 \\
\hline & & 30 & 20.03 & 19.46 & 20.12 & 10.50 & 19.35 & 5.70 \\
\hline & \multirow[t]{4}{*}{50} & 8 & 11.16 & 34.51 & 13.65 & 19.35 & 14.44 & 11.23 \\
\hline & & 12 & 10.90 & 24.88 & 14.00 & 12.10 & 14.05 & 7.32 \\
\hline & & 20 & 11.09 & 42.37 & 13.78 & 18.74 & 15.04 & 11.48 \\
\hline & & 30 & 13.46 & 21.14 & 15.66 & 7.29 & 16.85 & 3.15 \\
\hline & \multirow[t]{4}{*}{100} & 8 & 7.24 & 43.10 & 9.59 & 21.59 & 11.69 & 10.70 \\
\hline & & 12 & 6.95 & 27.20 & 10.70 & 11.57 & 11.85 & 6.51 \\
\hline & & 20 & 7.87 & 46.91 & 10.59 & 21.43 & 12.79 & 13.46 \\
\hline & & 30 & 9.17 & 19.87 & 12.30 & 4.65 & 12.83 & 2.63 \\
\hline \multirow[t]{8}{*}{50} & \multirow[t]{4}{*}{50} & 8 & 15.26 & 51.81 & 18.47 & 29.33 & 18.54 & 19.01 \\
\hline & & 12 & 12.50 & 48.98 & 15.61 & 26.68 & 17.17 & 18.62 \\
\hline & & 20 & 11.70 & 60.20 & 15.09 & 36.23 & 15.20 & 22.96 \\
\hline & & 30 & 14.27 & 37.70 & 18.38 & 21.41 & 31.35 & 5.54 \\
\hline & \multirow[t]{4}{*}{100} & 8 & 10.98 & 64.20 & 13.71 & 30.27 & 15.59 & 19.19 \\
\hline & & 12 & 10.43 & 47.46 & 13.72 & 25.62 & 15.86 & 14.18 \\
\hline & & 20 & 10.24 & 69.49 & 13.42 & 34.47 & 30.33 & 10.14 \\
\hline & & 30 & 13.21 & 36.98 & 27.49 & 8.38 & 35.00 & -4.16 \\
\hline
\end{tabular}

instances with 50 origins, 100 destinations, 30 scenarios and $2 / 3$ commodities. In any case, it becomes clear that, when considering the uncapacitated case, a general solver seems to provide an efficient tool for solving or, at least, for approximating quite accurately, the optimal solution to the problem.

On the other hand, for the capacitated case, very few instances could be solved successfully to optimality using the solver. In general, the solver could not obtain 
an actual optimal solution within the time limit (note that in this table we do not include the column 'CPUmax' because the corresponding values are always equal to the time limit). This is not surprising as we are dealing with a mixed 0-1 stochastic programming problem and thus a problem that can be expected to be hard to solve (Escudero 2009).

Nevertheless, it should be pointed out that in the majority of instances for which an optimal solution was not obtained within the time limit, the final gap is quite small, which indicates that a good approximation to the optimal solution can, in general, be obtained within the time limit. The exception to this regards the instances with 50 origins and 3 commodities with 20 and 30 scenarios.

Another aspect that can be observed in Tables 2 and 3 regards the slight increase of the gaps when the number of commodities and/or the number of scenarios increases. Note, however, that the increase is small. This was expected. In fact, this behavior simply translates the increase in the dimension of the instances which, often, makes a mixed 0-1 problem harder to solve.

In Tables 4 and 5, we can observe the relative percentage VSS and the cpu time (in seconds) required to obtain such value for the uncapacitated and capacitated problems, respectively. An entry in the column headed by ' $\mathrm{VSS}_{R}$ ' gives the average relative percentage value (\%) of the stochastic solution as introduced in Sect. 3.3. Note than in our case, $p_{s}=\frac{1}{|S|}, s \in S$. An entry in the columns headed by 'CPU' corresponds to the average cpu time (seconds) required by the solver to obtain the optimal/best value of the stochastic solution. As before, the symbol "-_" indicates that the time limit was reached for the computations required by the five instances corresponding to the entry. It should be noted that when the time limit is reached, we consider the value of the best integer solution found in the computation of $V S S_{R}$.

The observation of Tables 4 and 5 gives a first indication on the relevance of using a stochastic approach for the problem we are addressing. For the uncapacitated case (Table 4) we realize that the first-stage solution provided by the expected value problem leads to an accurate approximation for the optimal first-stage decision. However, this is far from true in the capacitated case. In fact, observing Table 5, we realize that the relative $V S S$ is quite significant in most of the instances.

Table 4 allows us to conclude that the relevance of using a stochastic approach is marginal for the uncapacitated case. Accordingly, we proceed the analysis only for the capacitated problem. For this case, Table 6 reports the relative values $\operatorname{EEV}_{R}$ and $\mathrm{EVPI}_{R}$ discussed in Sect. 3.3. Note, again, that in our case $p_{s}=\frac{1}{|S|}, s \in S$.

The observation of Table 6 gives strength to the relevance of using a stochastic approach for the problem we are addressing. In fact, the percentage values are quite significant across all the instances. It is also important to note that the GAP of the stochastic problem gets larger as the instance size increases. Thus, since the best feasible solution provided by the solver is the value considered for producing the values in columns $\mathrm{VSS}_{R}$ and $\mathrm{EEV}_{R}$, the decrease in these values is naturally expected. In addition, this same phenomenon explains the negative value that appears in Tables 5 and 6. Such negative value simply means that the solver was not able to find a feasible solution better than the solution provided by the expected value problem, which shows the difficulty of the problem at hand. 
As far as the $\mathrm{EVPI}_{R}$ is concerned, we realize that the difference between the values of the solution induced by the stochastic problem across all scenarios is quite different from the value we would obtain with perfect information. This gives an indication that the "degree of stochasticity" in the data is quite significant, which fully justifies the use of a stochastic approach. As far as the $\mathrm{EEV}_{R}$ values are concerned, we conclude that, again, the average difference across all scenarios between the solution induced by the expected value problem and the stochastic solution is again quite significant. This is a clear indication that considering the expected value problem for approximating the first-stage optimal solution is an oversimplification for the problem at hand.

\section{Conclusion}

In this paper, a two-stage stochastic transportation problem was considered that comprises fixed handling costs at the origins and fixed set-up costs for the transportation channels. The problem was formulated as a two-stage stochastic mixed 0-1 programming problem. In the first stage, a decision is made about the links to select. In the second stage, a recourse decision is considered for the amounts to ship and the origins to activate. The problem was presented in its simpler setting and was progressively extended in order to accommodate multiple commodities and capacities at the origins.

Based on the results provided by a series of computational tests performed, we conclude that like in other optimization problems, the inclusion of capacity constraints increases dramatically the difficulty of the problem and makes it significantly harder when it comes to solving the problem to optimality using a general solver. Furthermore, the relevance of considering a stochastic approach is negligible in the uncapacitated case but it is quite significant in the capacitated one. Therefore, in the latter case, it is definitely worth considering a modeling framework which captures the uncertainty in the data.

This paper opens several research directions. One concerns the need for the development of exact and approximate procedures specifically tailored for the capacitated problem. Another aspect worth investigating regards possible extensions to the problem addressed in this paper as it is the case in which there are specific link capacities. In such case, as it happened when capacities were considered at the origins, the stochastic problem does not have relatively complete recourse and the first-stage feasibility must be properly accounted for.

Acknowledgments This research has been partially supported by projects FQM-5849 (Junta de Andalucía $\backslash$ FEDER) and MTM2010-19576-C02-01 (MICINN, Spain) and by the Portuguese Science Foundation-Centro de Investigação Operacional. The authors would like to express their gratitude to the anonymous referees for the constructive comments and suggestions given, which helped to improve the paper.

\section{References}

Ahuja RK, Magnanti TL, Orlin JB (1993) Network flows: theory, algorithms, and applications. Prentice Hall, London 
Barbarosoğlu G, Arda Y (2004) A two-stage stochastic programming framework for transportation planning in disaster response. J Oper Res Soc 55:43-53

Birge JR (1982) The value of the stochastic solution in stochastic linear programs with fixed recourse. Math Prog 24:314-325

Birge JR, Louveaux F (1997) Introduction to stochastic programming. Springer, London

Escudero LF (2009) On a mixture of the fix-and-relax coordination and lagrangian substitution schemes for multistage stochastic mixed integer programming. TOP 17:5-29

Escudero LF, Garín A, Merino M, Prezé G (2007) The value of the stochastic solution in multistage problems. TOP 15:48-64

Escudero LF, Garín MA, Pérez G, Unzueta A (2012) Lagrangian decomposition for large-scale two-stage stochastic mixed 0-1 problems. TOP 20:347-374

França PM, Luna HPL (1982) Solving stochastic transportation-location problems by generalized Benders decomposition. Transp Sci 16(2):113-126

Grieco S, Semeraro U, Tolio T (2001) A review of different approaches to the fms loading problem. Int J Flexible Manuf Syst 13(4):361-384

Holmberg K (1995) Efficient decomposition and linearization methods for the stochastic transportation problem. Comput Optim Appl 4:293-316

Holmberg K, Jörnsten KO (1984) Cross decomposition applied to the stochastic transportation problem. Eur J Oper Res 17(3)

Holmberg K, Tuy H (1999) A production-transportation problem with stochastic demand and concave production costs. Math Prog 85:157-179

LeBlanc LJ (1977) A heuristic approach for large scale discrete stochastic transportation-location problems. Computers Math Appl 3:87-94

Li ACY, Nozick L, Xu N, Davidson R (2012) Shelter location and transportation planning under hurricane conditions. Transp Res Part E: Logistics Transp Rev 48:715-729

Lium A-G, Crainic TG, Wallace SW (2009) A study of demand stochasticity in service network design. Transp Sci 43:144-157

Max Shen Z-J, Coullard C, Daskin MS (2003) Joint location-inventory model. Transp Sci 37(1):40-55

Qi L (1985) Forest iteration method for stochastic transportation problem. Math Prog Study 25:142-163

Thapalia BK, Crainic TG, Kaut M, Wallace SW (2012a) Single-commodity network design with random edge capacities. Eur J Oper Res 220:394-403

Thapalia BK, Crainic TG, Kaut M, Wallace SW (2012b) Single-commodity stochastic network design with multiple sources and sinks. INFOR 49:193-211

Tsai M-T, Saphores J-D, Regan A (2011) Valuation of freight transportation contracts under uncertainty. Transp Res Part E: Logistics Transp Rev 47:920-932

Williams AC (1963) A stochastic transportation problem. Oper Res 11(5):759-770

Xu N, Nozick L (2009) Modeling supplier selection and the use of option contracts for global supply chain design. Computers Oper Res 36:2786-2800 